\title{
Insights on the Fractal-Fracture Behaviour Relationship
}

\author{
José de Anchieta Rodrigues, Victor Carlos Pandolfelli
}

\author{
Departamento de Engenharia de Materiais, \\ Universidade Federal de São Carlos, \\ Via Washington Luiz, km 235, 13.565-905 São Carlos - SP, Brazil; \\ e-mail: josear@power.ufscar.br
}

Received: March 5, 1998; Revised: August 15, 1998

\begin{abstract}
The fractals theory has been increasingly applied in the field of materials science and engineering. Models of fractal lines and surfaces have been generated to describe the microstructural features of materials. Special interest is placed upon a description of the fracture surface based on a fractal geometry in order to understand the crack path in materials. Several papers have demonstrated the relationship between the fractal dimension of a fracture surface and the values of roughness and fracture toughness. In this work an extension of the theory of fractals for ceramic materials is proposed, to which the crack deflection toughening mechanism is thought to be related. In order to accomplish this objective, a review describing the concept of fractals and its relationship with the fracture toughness is presented. In the following part, a correlation between fractal dimension, total energy of fracture and the average resistance to crack propagation is proposed; all these parameters being dependent on the history and on the complexity of crack propagation path.
\end{abstract}

Keywords: fractals, ceramics, fracture surface, total energy of fracture, $R$-curve

\section{Introduction}

The importance of overcoming problems related to the brittleness of ceramic materials has been constantly emphasized in the technical literature. In the last two decades several toughening mechanisms have been designed, making the ceramics much more appropriate to certain practical applications, namely those which require thermal shock resistance. The most common toughening mechanisms include microcracking branching, crack deflection and stress induced phase transformation.

Advances in understanding the fracture process has been possible through fractographic techniques. Equally important is to find out new characterization techniques to evaluate brittleness, which could provide information on its origin. As pointed out by Lin and $\mathrm{Lai}^{1}$, in 1977 Mandelbrot proposed a new characterization technique for fracture surfaces applying fractal theory concepts.

This new concept was extended by Hornbogen ${ }^{2}$, who published a review on the use of fractal structure to describe microstructural features of metals relating a large number of parameters, such as the distribution of phases, martensitic and dendritic configuration, dislocation arrays, slip bands, grain boundaries, and fracture surface roughness.
There are several works in the literature which investigate the relationship between fractal dimension of a fracture surface and experimental values of the corresponding fracture toughness, $\mathbf{K}_{\text {Ic }}$, following suggestions of Mandelbrot, Passoja and Paullay ${ }^{3}$. The most frequent properties and features reported in these works are impact energy ${ }^{3}$, fracture toughness ${ }^{1,4-6}$, type of fracture along the crack propagation $^{7}$ and roughness 8 .

Basic relationships found in the literature are shown in this paper. An extension of the fractal theory for ceramic materials which predominantly display geometric toughening mechanisms (crack deflection) is proposed, based on relationships between fractal dimension and total energy of fracture, as well as on the average value of the crack propagation resistance.

\section{Concepts of Fractals}

The fractal geometry is a mathematical concept that describes objects of irregular shape ${ }^{9}$. Some natural geometrical shapes, that can be irregular, tortuous, rough or fragmented, can be described using concepts of fractal geometry $^{3}$ as long as the requirement of self-similarity is satisfied. The latter term implies that the geometrical fea- 
tures of an object are independent of the magnification or observation scale.

An artificial manner to generate a fractal line comprises application of a known criterion, such that of the Koch's line $^{2,9}$. It is useful to simulate a progressive tortuosity in a straight line as a mean to model a crack propagation path in a brittle material ${ }^{6}$.

The structure of Koch's line is based on a figure constituted of four straight segments of same length $\left(\mathbf{L}_{\mathrm{s} 1}=(1 / 3) \mathbf{L}_{\mathrm{p}}\right)$. This figure defines an object whose projected length equals $\mathbf{L}_{\mathrm{p}}$, as shown at the top of Fig. 1. The first object that is formed is not flat. Instead, it exhibits a low degree of tortuosity described by its hilly profile. In a recurrent manner it is possible to increase the tortuosity on the original line by adding new smaller straight segments, repeating the same picture, but progressively in a smaller scale.

Let us establish a reduction index $n=1 / 3$ and designate $\mathrm{x}$ as the number of applied reductions (iterations). The

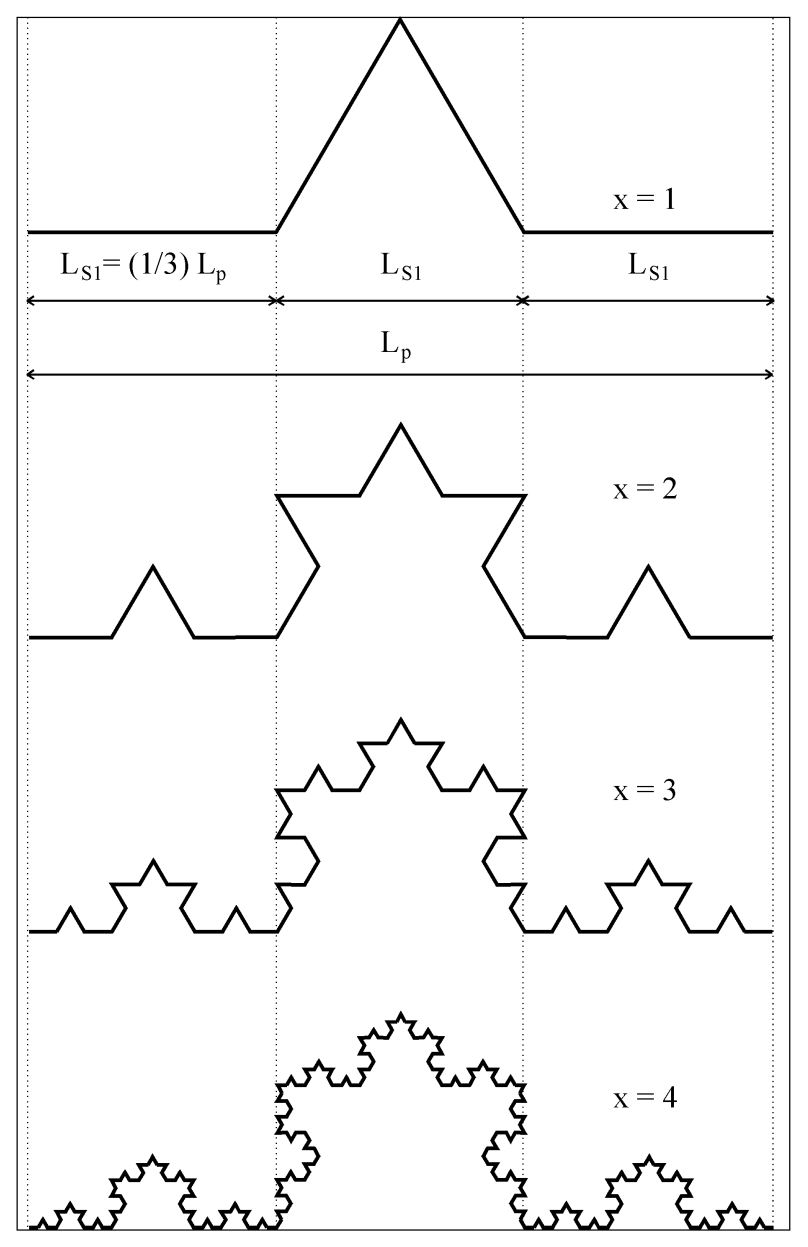

Figure 1. Koch's line showing different degrees of iteration. The line at the top highlights the shape of the basic picture constituted of four straight segments. bottom part of Fig. 1 shows the aspect of Koch's line after four iterations $(\mathrm{x}=4)$.

An important consequence of the geometry of a fractal line is that its true length depends on the size of the measuring ruler. It is possible to demonstrate that, after each iteration, the total true length of the line increases in accordance with the following relation

$$
\mathrm{L}_{\mathrm{x}+1}=\mathrm{L}_{\mathrm{x}} \mathrm{N} \mathrm{n}
$$

where $\mathrm{x}$ is the number of iterations, $\mathrm{N}$ is the number of elements in the basic picture $(x=1)$ and $n$ is the applied reduction. Thus, the true length of a fractal line after $\mathrm{x}$ iterations is given by:

$$
\mathrm{L}_{\mathrm{x}}=\mathrm{L}_{\mathrm{p}} \varepsilon_{\mathrm{x}}^{(\mathrm{d}-\mathrm{D})}
$$

where $\varepsilon_{\mathrm{x}}=\mathrm{n}^{\mathrm{x}}$ represents the fractional measuring unit, which, for practical purposes, will be associated either to the magnification and resolution used in the microscope ${ }^{2}$ or to the relative length of a typical constructional segment of that line ${ }^{5,6}$. The parameter $d$ represents the Euclidean dimension of the object ( $d$ is equal to 1 for a line or equal to 2 for a surface) and D is denominated fractal dimension. Lines totally contained in a plane display D-values between 1 and 2 . The higher the D-value the greater will be the tortuosity of the line.

The parameter $\varepsilon_{\mathrm{x}}$ has two important meanings. Firstly, it can represent the size of the measuring ruler. However it is known that for real objects; a fracture surface, for instance; its fractal features are limited between minimum and maximum bounds. For brittle materials, it is assumed that the minimum $\varepsilon_{\mathrm{x}}$-value is associated to the atomic spacing and the grain size to the maximum one ${ }^{6}$. Secondly, $\varepsilon_{\mathrm{x}}$ can be represented by a segment or an area, which are typical features of a fracture surface. This later assumption leads to equations relating properties and fractal dimension, as shown in the following sections.

The following equation, which expresses the general definition of fractal dimension, can be used to work out the dimension of an artificial fractal object,

$$
\mathrm{D}=\frac{\log (\mathrm{N})}{\log (1 / \mathrm{n})}
$$

Applying this equation to Koch's line, assuming $\mathrm{N}=4$ and $n=1 / 3$, the D-value will be equal to 1.2618 .

The same approach can be used to treat a surface. Starting at an Euclidean dimension equal to 2, fractal dimensions greater than this value will represent rough surfaces, similar to the fracture surface of polycrystalline ceramics. As a reference, either the cleavage of a single crystal or the fracture of a glass can generate a plain fracture surface with $\mathrm{D}=2.0$, when observed in a microscopic scale. In fact, cleavage steps will be found in a nanoscale. 


\section{Determination of Fractal Dimension of Fracture Surfaces}

One of the most commonly employed techniques for determination of fractal dimension of a fracture surface was introduced by Mandelbrot et al. ${ }^{3}$. In this method, named the "slit island method", the fractured specimens are firstly mounted in resin. The following step consists of careful polishing of the surface parallel to the fracture surface in order to highlight the ceramic "islands". The island structure has a correlation with the relief of the fracture surface topography. A schematic view of this procedure is shown in Fig. 2. For each polishing depth the enclosed areas of the islands are measured and plotted against the corresponding perimeter of the contour of the islands.

It is shown in the literature that the log-log plot of the measured area versus its corresponding perimeter, generates a straight line whose slope is related to the fractal dimension of the fracture surface. Therefore, it is possible to find out the correlation between the independently measured $\mathbf{K}_{\text {Ic }}$ parameter and the fractal dimension obtained by the slit island method.

Furthermore, it is possible to obtain D from Eq. 2 through measurements of sectional profiles of the crack surface, as illustrated in Fig. 3, after cutting the sample perpendicularly to the fracture surface or using an image analysis technique which is able to digitally track the profile. However one must be aware that slit island method and perpendicular profile analysis might generate different values for $\mathrm{D}$.

As an example ${ }^{10}$, a simulated profile represented by a Koch's line with $\mathbf{L}_{\mathrm{p}}=0.30 \mathrm{~m}$ and $\mathrm{x}=4$ was used. The true length of the line, $\mathbf{L}$, was measured using rulers of different sizes, equal to $\mathbf{L}_{\mathrm{p}}$ and to fractional values of this. The result is shown in a plot of relative length, $\mathbf{L} / \mathbf{L}_{\mathrm{p}}, v s$. the measuring unit, $\varepsilon_{\mathrm{x}}$, in Fig. 4. In a $\log -\log$ plot, a linear relation is obtained and the value of $\mathrm{D}$ is equal to 1.24 presenting a good agreement with the theoretical value for Koch's line.

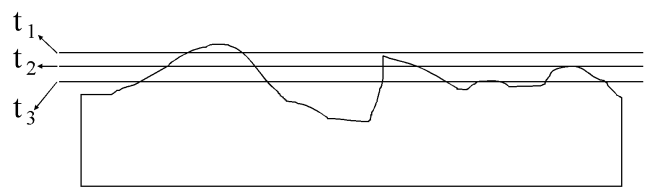

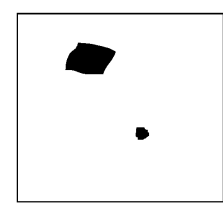

$\mathrm{t}_{1}$

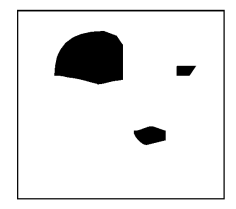

$\mathrm{t}_{2}$

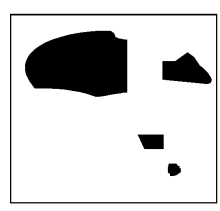

$\mathrm{t}_{3}$
Figure 2. Schematic view of the procedure employed to reveal the "slit island structure". $\mathrm{t}_{1}, \mathrm{t}_{2}$ and $\mathrm{t}_{3}$ represent the evolution of the slit islands for increasing polishing times.

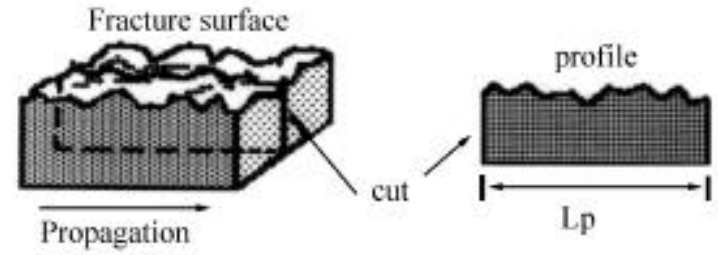

Figure 3. A fracture surface and its corresponding cutting profile.

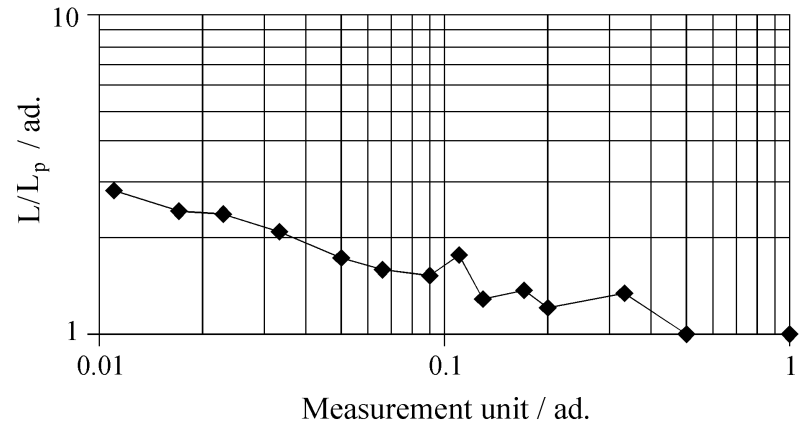

Figure 4. Relative true length versus the measurement unit for a Koch's line with $\mathrm{x}=4$ and $\mathbf{L}_{\mathrm{p}}=0,30 \mathrm{~m}$ (ad. = non-dimensional) ${ }^{10}$.

Also in Fig. 4, the line reveals some peaks that are related to the length of characteristic segments of Koch's line. This suggests that the method is able to indicate the characteristic lengths of a fracture surface which, in turn, can be related with microstructural features ${ }^{6}$.

\section{Fracture Aspects}

\subsection{General concepts}

Important basic relationships that describe the role of fractal theory for fracture can be found in works reported by Lin and Lai ${ }^{1}, \mathrm{Mu}$ and Lung ${ }^{5}$, Tanaka ${ }^{6}$, Xin et al. ${ }^{8}$, Nagahama $^{11}$ and Lung ${ }^{12}$.

For a fracture surface such as those observed in single crystals or in glass (which could be microscopically flat but not in a nanoscale), the driving force for crack extension can be represented by the intrinsic elastic energy release rate, $\mathbf{G}_{\text {Ico, }}$, and can be written as

$$
\mathbf{G}_{\text {Ico }}=2 \gamma_{\mathrm{o}}=\frac{\mathbf{K}_{\text {Ico }}^{2}}{\mathbf{E}^{\prime}}
$$

where $\gamma_{0}$ is the surface energy per unit area, expressing only the thermodynamic surface energy (in practice, the energy necessary to break chemical bonds). $\mathbf{K}_{\text {Ico }}$ is the plaine strain intrinsic fracture toughness for mode I of stressing and $\mathbf{E}$ ' is defined as $\mathbf{E} /\left(1-v^{2}\right)$, where $\mathbf{E}$ is the Young's modulus and $v$ is the Poisson's ratio of the material.

Nevertheless, at room temperature, even a ceramic material exhibits some microplasticity in the crack tip, which causes stress release. This contribution for the fracture energy is symbolized by $\gamma_{p}$. In this paper, it is assumed that 
$\gamma_{\mathrm{p}}$ is independent on the direction of crack propagation and can be defined per unit of fracture area. Therefore, effectively the crack propagation initiates when

$$
\mathbf{G}_{\text {Ic }}=2 \gamma_{\text {eff. }}=\frac{\mathbf{K}_{\text {Ic }}^{2}}{\mathbf{E}^{\prime}}
$$

where $\mathbf{G}_{\text {Ic }}$ is the effective elastic energy release rate, $\mathbf{K}_{\text {Ic }}$ is the fracture toughness (effective critical stress intensity factor) and $\gamma_{\text {eff. }}=\gamma_{\mathrm{o}}+\gamma_{\mathrm{p}}$. Usually, $\mathbf{K}_{\mathrm{Ic}}$ and, in turn, $\gamma_{\mathrm{eff} \text {. are }}$ experimentally measured through fracture testes under catastrophic crack propagation condition.

Back to Fig. 3 the resulting tortuous line (profile) on the fracture surface corresponds to crack path along the cut plane. Therefore, the more tortuous is the line (i.e. rougher fracture surface) the longer will be its true length, regarding to its constant projection $\mathbf{L}_{\mathrm{p}}$.

Recent papers do not present details on how to incorporate the effect of true area increase on $\gamma_{\text {eff., }}$ since they do not consider the concept of fractal structure. Davidge and Evans ${ }^{13}$ mentioned in their publication that the surface energy criteria for crack propagation must consider a number of effects, namely, plastic flow, the work needed to generate cleavage steps, the energy consumed in creating subsidiary cracks, and, obviously, the $\gamma_{0}$ itself. The referred researchers pointed out that $\gamma_{0}$ should be multiplied by a geometrical factor to account for the roughness of the fracture face, but no further efforts were done to express it in a quantitative way.

The description of the true path of a crack profile is obtained when $d=1$ is applied to Eq. 2. The same increasing factor in the true length of the crack path is also verified in the true area of the fracture surface. Let $\gamma_{0}$ be constant and equal to the average of different crystallographic planes in a certain crystal (as reference, it could also be thought in the fracture of a glass). For a larger true fracture area more energy for fracture will be consumed. Nevertheless, the area that is included in the practical calculation of the surface energy is the projected one. The consequence is that the resulting surface energy will be higher than $\gamma_{\text {eff. }}$ defined in conditions of Eq. 5. The surface energy calculated in this manner continues to be named "effective surface energy", but it includes a term related to the area increment due to tortuosity (or roughness).

Considering a situation where a ceramic material has only the contributions of $\gamma_{\mathrm{o}}, \gamma_{\mathrm{p}}$, and those due to the fractal nature as an additional microstructural mechanism, as shown bellow:

$$
\mathbf{G}_{\mathrm{Ic}}=2 \gamma_{\mathrm{eff} .} \approx 2\left(\gamma_{\mathrm{o}}+\gamma_{\mathrm{p}}\right) \varepsilon_{\mathrm{x}}^{(1-\mathrm{D})} \approx \frac{\mathbf{K}_{\mathrm{Ic}}^{2}}{\mathbf{E}^{\prime}}
$$

the greater the fractal dimension of the crack path profile (or of the fracture surface) the larger will be the $\mathbf{K}_{\mathrm{Ic}}$-value.
Equation 6 is a comprehensive representation of some ideas reported in the literature. Some authors use the equality sign to relate terms in the equation whereas some others use the proportionality sign $(\approx)$. In the opinion of the authors, the proportionality sign is more adequate, since the same increment factor of fracture area does not necessarily correspond to an increase of $\mathbf{K}_{\text {Ic }}^{2}$ and, consequently, of $\gamma_{\text {eff. }}$. The total increment of fracture area is associated with the total process of fracture, but $\mathbf{K}_{\mathrm{Ic}}$ is a property related only with the beginning of crack propagation under catastrophic condition. Another important point is that, rigorously speaking, Eq. 5 is somewhat imprecise when applied to rough fracture ${ }^{14}$.

In the simplest situation, roughness of fracture surface can have its origin during intergranular fracture. The latter consists of an intrinsic crack deflection mechanism that imposes a greater stress intensity factor for crack propagation initiation.

Still, in Eq. 6 it must be observed that the relationship between the two last terms is frequently verified in experiments with several different materials. Usually the plot of $\log \left(\mathbf{K}_{\mathrm{Ic}}\right)$ vs. D can be represented by a straight line ${ }^{5,6,8,11,15}$, confirming Eq. 6.

Based on Griffith-Orowan-Irwin relation $\left(\mathbf{G}_{\text {Ic }}=\left(\mathbf{K}_{\text {Ic }}^{2} / \mathbf{E}^{\prime}\right)=2 \gamma_{\text {eff. }}\right)$, which is valid for flat fracture surfaces, Nagahama ${ }^{11}$ showed that the dependence of $\mathbf{K}_{\mathrm{Ic}}$ on $\mathrm{D}$ can be predicted from the degree of brittleness of the tested material. Therefore, it has been found ${ }^{11}$ that $\mathbf{K}_{\mathrm{Ic}}$ decreases with the increase of D for ductile materials, whereas for brittle materials $\mathbf{K}_{\text {Ic }}$ scales with D. Equation 6 establishes a new insight ${ }^{11}$ in which fracture occurs when the elastic energy release rate is greater than the effective energy of fracture corrected by the fractal effect.

\subsection{The roughness of the fracture surface}

Taking into account a flat fracture surface, such as a single crystal cleavage, it is correct to assume $\gamma_{\text {eff. }}=\gamma_{\mathrm{o}}+\gamma_{\mathrm{p}}$, where $\gamma_{p}$ is related to the contribution of possible microplasticity that can occur in the frontal zone of a crack in a ceramic material. Considering a brittle polycrystalline ce-

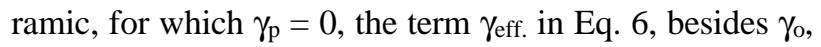
should be also associated to the fact that real fracture surface is rough and therefore, it also affects crack propagation initiation. It must also be emphasized that $\gamma_{p}$ markedly depends on the temperature.

Roughness is a fracture surface parameter that has a clear physical meaning. Based on Xin et al. work $^{8}$, rough- $^{-}$ ness can be defined as the ratio $\mathbf{Q}$ between the real area of fracture surface, $\mathbf{A}_{\mathrm{r}}$, and the projected area of fracture, $\mathbf{A}_{\mathrm{p}}$. Therefore, similarly to Eq. 2 it is possible to write this relation as 


$$
\mathbf{Q}=\frac{\mathbf{A}_{\mathrm{r}}\left(\varepsilon_{\mathrm{x}}\right)}{\mathbf{A}_{\mathrm{p}}}=\varepsilon_{\mathrm{x}}^{(2-\mathrm{D})}
$$

which establishes a relationship between roughness and fractal dimension. Compared with Eq. 2, the number 2 in the above equation replaces the number 1 , since the Euclidean dimension of a smooth surface is equal 2 . Consequently, the value of $\mathrm{D}$ is equal, or greater than 2 in Eq. 7.

As previously noted, Eq. 5 is valid for fracture along a flat surface. Consider now a fracture that generates a rough surface (polycrystalline ceramics, for instance), in which the surface energy per unit area of projected surface is $\gamma_{\text {eff. }}$. Then, combining Eqs. 5, 6 and 7 it is possible to write:

$$
\begin{aligned}
& \frac{\mathbf{G}_{\text {Ic, rough }}}{\mathbf{G}_{\text {Ic, flat }}}=\frac{\mathbf{K}_{\text {Ic, rough }}^{2}}{\mathbf{K}_{\text {Ic, flat }}^{2}}=\frac{\gamma_{\text {eff., rough }}}{\gamma_{\text {eff., flat }}} \approx \\
& \frac{\left(\gamma_{0}+\gamma_{\mathrm{p}}\right)_{(\mathrm{x}}^{(2-D)}}{\left(\gamma_{\mathrm{o}}+\gamma_{\mathrm{p}}\right)}=\mathbf{Q}=\frac{\mathbf{A}_{\mathbf{r}}}{\mathbf{A}_{\mathbf{p}}}=\varepsilon_{\mathrm{x}}^{(2-\mathrm{D})}
\end{aligned}
$$

Equation 8 summarizes the main relationships found in the literature.

\subsection{The new proposal}

The fractal dimension expresses the fracture surface roughness and also represents the complexity of the crack's path. Since the total energy of fracture, $\gamma_{\text {wof., }}$ represents the average difficulty for crack propagation (in $\mathrm{J} / \mathrm{m}^{2}$ ), it must also be associated with the fractal dimension. This proposition is more adequate for ceramic materials that display purely geometrical toughening mechanisms, since $\gamma_{\text {wof. }}$ brings into consideration the history of stable crack propagation, whereas $\gamma_{\text {eff. }}$ is related to the beginning of catastrophic crack propagation.

Pezzotti et al. ${ }^{16}$ carried out an interesting work which established an experimental correlation between $\mathbf{K}_{\mathrm{Ic}}, \gamma_{\mathrm{wof}}$ and a fractal parameter for hot isostatically pressed $\mathrm{Si}_{3} \mathrm{~N}_{4} / \mathrm{SiC}$ composites. Using a profilometric analysis of the fracture surface, the authors generated log-log plots relating linear roughness of surface profiles to the measuring unit. The resulting curves are sigmoidal-like and the corresponding fractal parameter can be extracted from the slope. The observed changes in $\mathbf{K}_{\mathrm{Ic}}, \gamma_{\mathrm{wof}}$ and in the fractal parameter were due to differences in the microstructures, which changed the average size of $\mathrm{SiC}$ platelets in a $\mathrm{Si}_{3} \mathrm{~N}_{4}$-matrix.

Another paper by Pezzotti et al. ${ }^{15}$, reports the work carried out with heat treated $\mathrm{Si}_{3} \mathrm{~N}_{4} / \mathrm{SiC}$ composites. It describes extensive efforts to obtain the R-curve of these composites. However, in this article, the authors do not quantitatively correlate any R-curve parameter to the fractal parameter.

Several other works have described important experimental correlations between $\mathbf{K}_{\mathrm{ic}}$ or $\gamma_{\mathrm{wof}}$ and a fractal pa- rameter, although without establishing direct relations between any of the fractal parameters and the R-curve ${ }^{17}$. The present work also correlates $\overline{\mathbf{R}}$ (average R-value of an $\mathrm{R}$-curve) and the fractal dimension.

It is known that the R-curve expresses the history of instantaneous difficulties for crack propagation. Pandolfelli et al. ${ }^{18}$ proposed that $\overline{\mathbf{R}}$, calculated over all points of an R-curve, should represent an average difficulty for crack propagation, therefore, $\mathbf{R}$ equals to $2 \gamma_{\text {wof. }}$ could be assumed.

The value of $\mathbf{R}$ is evaluated with basis on the mathematical definition of average, i.e.

$$
\mathbf{R}=\left(\frac{1}{\mathbf{a}-\mathbf{a}_{\mathbf{0}}}\right) \int_{\mathbf{a}_{0}}^{\mathbf{a}} \mathbf{R}(\mathbf{a}) \mathrm{d} \mathbf{a}
$$

where $\left(\mathbf{a}-\mathbf{a}_{0}\right)$ defines the integration interval for crack length, a. Equation 9 is applied on experimentally determined R-curves.

For ceramic materials where only geometrical toughening mechanisms are verified, it should be expected that the increase in total energy of fracture is simply due to the increase in true area of fracture surface. Hence, there would exist a correlation between $\mathbf{R}, \gamma_{\text {wof }}, \mathbf{Q}$, and D.

Assuming the additive concept of Davidge and Evans ${ }^{13}$, the following relation is proposed:

$$
\gamma_{\mathrm{wof}}=\gamma_{0}+\gamma_{\mathrm{p}}+\overline{\Delta \gamma}=\gamma_{\text {eff. }}+\overline{\Delta \gamma}
$$

which can be further expressed in the following form

$$
\gamma_{\mathrm{wof}}=\left(\gamma_{\mathrm{o}}+\gamma_{\mathrm{p}}\right)+\left(\gamma_{\mathrm{o}}+\gamma_{\mathrm{p}}\right)\left(\varepsilon_{\mathrm{x}}^{(2-\mathrm{D})}-1\right)
$$

In Eq. 10 a common notation $\overline{\Delta \gamma}$ was used to express a geometrical microstructural mechanism that contributes for toughness increment. Equation 11 refers to a straight crack path energetically represented by the term $\gamma_{\text {eff. }}=\left(\gamma_{0}+\gamma_{p}\right)$. In this equation, the fractal contribution of true surface area appears in $\overline{\Delta \gamma}$, which is written as $\left(\gamma_{0}+\gamma_{p}\right)$ $\left(\varepsilon_{\mathrm{x}}^{(2-D)}-1\right)$. It can be verified that for $\mathrm{D}=2$ (flat fracture surface) $\gamma_{\text {wof }}=\gamma_{\text {eff. }}$, since there is no microstructural contribution. This situation resembles a transgranular fracture or the fracture of a glass.

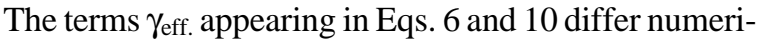
cally from each other, since the experimental procedure used to determine them are also distinct. Although, the reference states (smooth fracture surface) are the same for both Eqs. 6 and 10, the $\gamma_{\text {eff.-value in Eq. } 6 \text { is determined }}$ through $\mathbf{K}_{\mathrm{Ic}}$-tests, which require a catastrophic fracture condition. On the other hand, Eqs. 10 and 11 are associated to propagation under quasi-static crack extension.

Therefore, considering Eqs. 7 and 11, an extended proposition is given: 


$$
\mathbf{Q}=\frac{\mathbf{A}_{\mathrm{r}}}{\mathbf{A}_{\mathrm{p}}}=\frac{\gamma_{\text {wof }}}{\gamma_{\text {eff. }}}=\frac{\mathbf{R}}{\mathbf{R}_{\mathrm{o}}}=1+\frac{\overline{\Delta \gamma}}{\gamma_{\text {eff. }}}=\varepsilon_{\mathrm{x}}^{(2-\mathrm{D})}
$$

In Eq. 12, the reference is the average $\gamma_{\text {eff. }}$ either for all crystallographic cleavage planes of a single crystal or for fracture of the corresponding vitreous state. It is also implicit in Eq. 12 the acceptable assumption that $\mathbf{R}_{\mathrm{o}} \cong 2 \gamma_{\text {eff. }}$.

Experimental work is being carried out in order to verify the validity of this proposal and experimental results will be submitted shortly. Comparison of different microstructures in materials of identical chemical composition (amorphous and totally crystallized) might be used to validate Eq. 12, if interlocking and friction between grains could be minimized.

\section{Conclusions}

- It is pointed out the importance of concept of fractal structure in physical characterization of materials.

- The fractal dimension is closely related to roughness of the fracture surface and to mechanical properties such as fracture toughness.

- The fractal analysis can reveal characteristic lengths of a fracture surface and contribute to a better understanding of the influence of microstructure on fracture processes.

- It is proposed that the fractal dimension is related to $\gamma_{\text {wof }}$ and to $\overline{\mathbf{R}}$ when a purely geometrical toughening mechanism takes place.

\section{Acknowledgment}

The authors are grateful to CNPq and FAPESP for the support given to this work and to Dr. P. Sepulveda's comments.

\section{References}

1. Lin, G.M.; Lai, J.K.L. Fractal characterization of fracture surfaces in resin-based composite. J. Mat. Sci. Let., v. 12, p. 470-472, 1993.

2. Hornbogen, E. Fractals in microstructure of metals. Int. Mat. Rev., v. 34, n. 6, p. 277-296, 1989.

3. Mandelbrot, B.B.; Passoja, D.E.; Paullay, A.J. Fractal character of fracture surfaces of metals. Nature, v. 308, n. 19, April, p. 721-722, 1984.

4. Mecholsky, J.J.; Passoja, D.E.; Feinberg-Ringel, K.S. Quantitative analysis of brittle fracture surfaces using fractal geometry. J. Am. Ceram. Soc., v. 72, n. 1, p. 60-65, 1989.
5. Mu, Z.Q.; Lung, C.W. Studies on the fractal dimension and fracture toughness of steel. J. Phys. D: Appl. Phys., v. 21, p. 848-850, 1988.

6. Tanaka, M. Fracture toughness and crack morphology in indentation fracture of brittle materials. J. Mat. Sci., v. 31, p. 749-755, 1996.

7. Tsai, Y.L.; Mecholsky Jr, J.J. Fractal fracture of single crystal silicon. J. Mater. Res., v. 6, n. 6, p. 1248-1263, 1991.

8. Xin, Y.-B.; Hsia, K.J.; Lange, D.A. Quantitative characterization of the fracture surface of Si single crystals by confocal microscopy. J. Am. Ceram. Soc., v. 78, n. 12, p. 3201-3208, 1995.

9. Mandelbrot, B.B. The Fractal Geometry of Nature, Freeman, New York, p. 468, 1983.

10. Rodrigues, J.A.; Peret, C.M.; Pandolfelli, V.C. Relação entre energia total de fratura e dimensão fractal. Proceedings of $12^{\circ}$ CBECIMAT, Águas de Lindóia SP - Brazil, dezembro, 1996, v. 1, p. 373-376.

11. Nagahama, H. A fractal criterion for ductile and brittle fracture. J. Appl. Phys., v. 75, n. 6, p. 3220-3222, 1994.

12. Lung, C.W. Fractals and the fracture of cracked metals. Fractals in Physics, Pietronero, L.; Tosatti, E., eds., Elsevier Science Publ., p. 189-192, 1986.

13. Davidge, R.W.; Evans, A.G. The strength of ceramics: Review Paper, Mater. Sci. Eng., v. 6, p. 281-298, 1970.

14. Lei, W.; Chen, B. Fractal characterization of some fracture phenomena. Eng. Fract. Mech., v. 50, n. 2, 149-155, 1995.

15. Pezzotti, G.; Noda, K.; Okamoto, Y.; Nishida, T. Thermomechanically induced embrittlement in hot isostatically pressed $\mathrm{Si}_{3} \mathrm{~N}_{4} / \mathrm{SiC}$ composites. J. Mat. Sci., v. 28, p. 3080-3088, 1993.

16. Pezzotti, G.; Sakai, M.; Okamoto, Y.; Nishida, T. Fractal character of fracture surfaces and boundary values of toughness in a simple ceramic-ceramic system. Mat. Sci. Eng., v. A197, p. 109-112, 1995.

17. Russ, J.C. Examples of Fractal Surfaces, Ch.8 in Fractal Surfaces, Plenum Press, New York, p. 309, 1994.

18. Pandolfelli, V.C.; Nedochetko, A.P.; Silva, S.N.; Rodrigues, J.A.; Longo, E. Curva-R em refratários de MgO-C. Proceedings of $39^{\circ}$ Congr. Bras. de Cerâmica, Águas de Lindóia - SP, 10-13 Junho, v. 1, p. 516-521, 1995. 